\title{
High-speed digital visualization and high-frequency automated shock tracking in supersonic flows
}

\author{
Brenda H. Timmerman* \\ Andrew J. Skeen \\ Peter J. Bryanston-Cross \\ University of Warwick \\ Optical Engineering Laboratory \\ Coventry CV4 7AL United Kingdom

\section{P. G. Tucker ${ }^{\dagger}$ \\ R. J. Jefferson-Loveday \\ University of Wales} \\ Civil and Computational Engineering Centre \\ SA2 8PP Swansea, United Kingdom
}

\author{
James Paduano \\ G. R. Guenette, Jr. \\ Massachusetts Institute of Technology \\ Gas Turbine Laboratory \\ Cambridge, Massachusetts 02139
}

\begin{abstract}
A low-cost, robust, versatile digital shadowgraph visualization system is presented that provides a fast nonintrusive diagnostic for unsteady high-speed flows. The technique is particularly designed for realtime automated tracking of shock positions, enabling high-speed active shock control. The visualization system is based on a high-intensity white LED light source combined with a CMOS-imaging sensor, providing the system with three modes of operation: (1) high-resolution overall instantaneous visualization; (2) high-resolution visualization showing spatial-temporal variations in the flow field, allowing direct identification of areas where changes occur; (3) adjustable windowed visualization at reduced resolution at high frame rate (currently up to $980 \mathrm{~Hz}$ ). Experimental results are presented together with numerical simulations based on the high-accuracy NTS Navier-Stokes solver and Roe's flux difference splitting method. The flow studied is an adjustable underexpanded jet flow coming from a nozzle that is placed in a counterflowing Mach-2 flow. The interaction of the two flows results in a complex shock and expansion pattern, providing a challenging configuration for the numerical flow solver. By modulating the jet, high-frequency changes are induced in the interaction pattern, allowing simulation of shock movement in a supersonic inlet. Good correspondence between measured and numerical shock position and angle is found. () 2008 Society of Photo-Optical Instrumentation Engineers. [DOI: 10.1117/1.2992621]
\end{abstract}

Subject terms: shock tracking; shadowgraphy; CMOS; high-speed visualization; active shock control; compressible flow.

Paper 070934R received Dec. 4, 2007; revised manuscript received Aug. 5, 2008; accepted for publication Aug. 6, 2008; published online Oct. 29, 2008. This paper is a revision of a paper presented of the SPIE conference on Optical Diagnostics for Fluids, Solids, and Combustion II, August 2003, San Diego, California. The paper presented there appears (unreferred) in SPIE Proceedings Vol. 5191.

\section{Introduction}

A flow visualization system is presented, designed to provide a fast, low-cost, and easy-to-use diagnostic for aerodynamic design concepts. Besides providing a general flow visualization capability, it is designed specifically for tracking high-speed unsteady events in a supersonic flow.

The system was developed initially to be used in the design of an efficient supersonic inlet. A major design driver for supersonic diffusers is the requirement to prevent unwanted shock formation and shock blowout (supersonic unstart). One approach to increasing the efficiency of a gas turbine engine is to actively control the position of the intrapassage shock wave through feedback control, rather than traditional design methods.

\footnotetext{
*Address all correspondence to B. H. Timmerman, University of Warwick, School of Engineering, Optical Engineering Laboratory, Gibbet Hill Road, Coventry, CV4 7AL, UK; E-mail: bht@eng.warwick.ac.uk

${ }^{\dagger}$ Currently at: University of Cambridge, Whittle Laboratory, Cambridge CB3 0DY, United Kingdom.
}

0091-3286/2008/\$25.00 @ 2008 SPIE
To aid in the design and to study the dynamics and control of shock formation and movement, high-speed tracking of shocks is needed. This typically requires a positional shock measurement resolution better than $0.2 \mathrm{~mm}$ and temporal resolution in the millisecond range. Furthermore, to allow feedback control in these type of flows, the positional data need to be transmitted to an active flow control device at a rate in the region of $1 \mathrm{kHz}$.

The measurement technique developed here for dynamical shockwave tracking is based on a low-cost, robust digital shadowgraphy assembly. The developed system provides both a global visualization needed for initial determination of the approximate shock position, as well as a high-speed tracking of the shock-wave position at rates up to $1 \mathrm{kHz}$ based on a zoomed view of the shock region. Because of the unsteadiness in the flow, individual images need to be recorded at relatively short exposure times (microseconds), in order to freeze the instantaneous events and avoid motion blurring.

Shadowgraphy, as a well-established technique for compressible-flow-field visualization (see, e.g., Refs. 1 and 2 ), especially in visualizing shock waves, was chosen as a basis in order to build a robust system with a minimal num- 
ber of optical components. Furthermore, the use of a focused shadowgraphy system allows accurate determination of the actual position of shock waves in the flow.

The digital recording of the shadowgraph images allows direct processing, in this case used for online, real-time, continuous determination of the shock-wave position. This real-time information can then be used for direct-control feedback, e.g., to change flow settings in order to control the shock formation in a supersonic inlet.

Several high-speed flow visualization systems have been reported in the literature. For a long time, however, high frame rates could generally only be achieved by the use of specialized systems such as drum cameras. ${ }^{3,4}$ Direct digital imaging, however, has often been limited to recording only a few images at the high rate, ${ }^{5-7}$ impeding (e.g.) the extraction of high-frequency flow oscillations. Longer sequences have been obtained at high resolution using (expensive) dedicated cameras (e.g., Ref. 8). More recently, high-speed cameras have become available, allowing frame rates well beyond $1000 \mathrm{frames} / \mathrm{s}$ at megapixel resolution (e.g., Photron's Fastcam SA-1) rather than the 25 or $30 \mathrm{~Hz}$ of standard cameras. Although these systems have been fairly widely used in (e.g.) diesel spray analysis (e.g., Ref. 9), such cameras are still relatively expensive. Alternatively, high-speed tracking has been achieved using lower-cost photodiode systems (e.g., Ref. 10); however, these 1-D systems will only give information on a single point rather than an area in the flow.

In the setup presented here, a relatively low-cost CMOStype camera is used. The camera used here does not have a global electronic shutter; here it is used to allow a new type of imaging, spatial-temporal streak imaging, providing information on both temporal and spatial variation in a single image.

The described system shows a novel direct method to provide active shock control within a transonic test facility. The system was tested on an aspirated cone-shaped nozzle placed in a supersonic flow in the supersonic wind tunnel of the MIT Gas Turbine Laboratory. By varying the jet flow generated by the nozzle, the bow shock around the cone was modulated, generating a high-frequency oscillation of the bow shock.

The measured results are compared with results from axisymmetric numerical simulations made with a highaccuracy compressible flow solver ${ }^{11}$ run in parallel processor mode with a dual block grid.

\section{High-Speed Imaging Using a CMOS Camera}

The detection sensor used here is a Vitana PixeLINK PLA653 megapixel CMOS camera. The $\frac{1}{2}$-in. monochrome sensor has a full resolution of $1280 \times 1024$ pixels, and can be subwindowed down to $32 \times 8$ pixels at 8 -or 10 -bit depth. Moreover, the position of these windows can be chosen arbitrarily. The maximum frame-rate depends on the size of the chosen window, the full-resolution frame rate is 12 frames/s at 8-bit image depth. Furthermore it provides a single-cable FireWire connection, reading the image data directly into the memory of the host computer (400 Mbit/s), eliminating the need for a data acquisition card.

An essential feature of this camera is that the sensor lacks a global electronic shutter. The pixels in the sensor do not capture data at the same time, but rather are exposed pixel by pixel, row by row. As a consequence, the images that are captured are not instantaneous, but show a time delay between the rows. The start of each pixel's integration is staggered by the reciprocal of the pixel clock rate (up to $24 \mathrm{MHz}$ ). When operating the camera at full-frame resolution, this results in adjacent rows depicting events approximately $80 \mu$ s apart.

By changing the camera settings it is possible to operate the visualization system in three separate modes: still view mode, spatial-temporal streak view mode, and tracking view mode. Each of these modes produces a different type of result.

\subsection{Spatial-Temporal Streak View}

Running the camera in video mode results in images akin to those from a streak camera, i.e., the time at which the event is captured varies along the rows of the picture. Unlike that in a streak camera, however, the captured image is not a one-dimensional strip, but the whole two-dimensional area under investigation, i.e., each pixel in the image represents a different location as well as a different moment in time. The authors have therefore dubbed the result a spatialtemporal streak image, and have found it to be a useful way to visualize, in real time, the movement of shock waves that are oscillating at a speed that is otherwise difficult to capture.

Despite the fact that images are not taken instantaneously in this mode, the effect of motion blur in the images that are captured is limited to extremely high-speed events, and in general the data do not suffer from motion blur. In the flows studied in this paper, with the camera positioned so that the shock waves run primarily along the vertical axis of the image, the shock typically oscillates no more than \pm 10 pixels side to side. At full frame resolution, the sensor takes $1.2 \mu$ s to sample 20 adjacent pixels, meaning that a shock would have to oscillate at more than $20 \mathrm{kHz}$ before the readout rate caused rows to become motion-blurred by more than 1 pixel. While each row can therefore be considered an instantaneous view, adjacent rows depict the shock position separated by approximately $80 \mu \mathrm{s}$, with the bottom of the picture representing the most recent position. The result is a wobbly image, updated at 12 frames/s, that clearly shows the amplitude and frequency of shock movement.

\subsection{Still View Mode}

Without a global electronic shutter the camera is unable to capture an image across the entire sensor instantaneously, resulting in the spatial-temporal images described. To nevertheless obtain instantaneous snapshots, a shutter is in effect simulated by setting the camera to integrate for a time longer than the readout period of the whole sensor (i.e., the pixels capture information for a time longer than the readout period) combined with the exposure being determined by a flashing light source, illuminating the imaged scene for a controllable period. At the point when all rows are integrating, the light source is flashed for the desired exposure time. Thus, all pixels are exposed during the same "instant" (the duration of the flash), and a freeze-frame still image is obtained. This furthermore requires the timing of the flash 


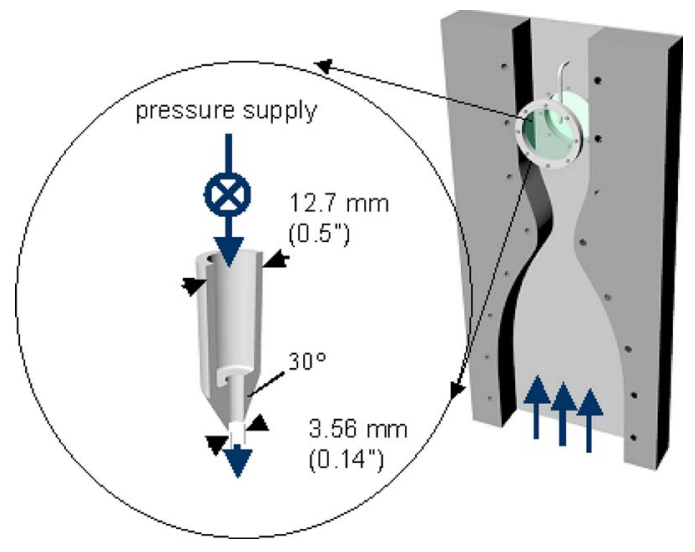

Fig. 1 Aspirated nozzle in supersonic wind tunnel.

to be accurately controlled. The PL-A653 camera provides TTL-level connections to facilitate the control of external flash and shutter mechanisms, but only down to pulses of $\sim 100 \mu$ s. Therefore, in the experiments described here, an external triggered pulse generator is used, allowing the light source (an LED in this case) to be pulsed for periods much shorter than the limit imposed by the camera. In the still-view-mode results shown in this paper, the pulse duration was $10 \pm 0.2 \mu \mathrm{s}$.

Still view mode therefore provides a live stream of instantaneously captured images. While theoretically limited to half of the normal frame rate (i.e., 6 frames/s for fullframe images), the implemented system ran at 2 frames/s because the images had to be stored on hard disk, rather than in RAM.

\subsection{Tracking View}

The CMOS sensor can be set to any subwindow size at any position. For a reduced number of pixels, the frame rate increases. At the maximum horizontal and minimum vertical resolution of $1280 \times 8$, the frame rate is typically 980 frames/s, with a readout period of approximately $640 \mu \mathrm{s}$. To reduce the readout time for the results shown here, only the last four rows of this subwindow were considered, resulting in an acquisition time of $320 \mu \mathrm{s}$, or approximately $1 / 8$ of a $400-\mathrm{Hz}$ oscillation period (for a negligible exposure period). A smaller horizontal resolution further reduces the acquisition time, meaning that for the purposes of tracking, the acquisition can be considered to be instantaneous.

\section{Flow Configuration}

The characteristics of unsteady shock behavior expected in supersonic inlets were simulated in a supersonic wind tunnel at the Gas Turbine Laboratory of MIT. The test section of this tunnel is vertical, with the flow direction upwards, and measures $203 \times 203 \mathrm{~mm}^{2}(8 \times 8 \mathrm{in}$.). Optical access is provided by two circular windows with a diameter of $133 \mathrm{~mm}\left(5 \frac{1}{4} \mathrm{in}\right.$.) in the sidewalls. An aspirated nozzle, shown in Fig. 1, was placed at the center of the test section, pointing downwards, i.e., in the direction opposite to the tunnel flow. The exit of this nozzle was placed at the level of the center of the optical access windows.

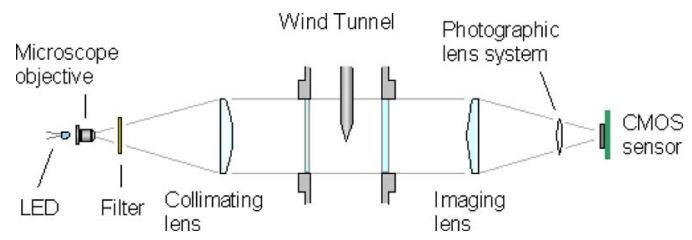

Fig. 2 Focused shadowgraphy system.

Air can be blown through the nozzle, generating an underexpanded free jet flow. With this configuration in the wind tunnel, different types of flow can be studied: firstly, the wind-tunnel flow around the nozzle, generating a steady shock-wave pattern around the spike; secondly, the free jet flow from the nozzle, generating a shock-diamond pattern typical of underexpanded free jets; and thirdly, a combination of the two. To simulate unsteady shock movement, the jet flow coming from the nozzle can be modulated by varying the nozzle pressure using a simple rotary valve. Thus, the diamond-shock pattern can be modulated, and more interestingly, the position of the bow shock in front of the nozzle in the jet-tunnel flow configuration can be varied, allowing testing of high-speed shock tracking. In the results shown here, the total pressure for the jet flow was varied to generate a jet flow at Mach number varying between approximately $M_{j}=0.9(1.7 \mathrm{bar})$ and $M_{j}=1.5$ (3.7 bar).

The tunnel parameters for the results presented are as follows:

Mach number $M_{t} 2.06$

Total pressure $105.5 \mathrm{kPa}$

Static pressure $11.2 \mathrm{kPa}$

Total temperature $311 \mathrm{~K}$

\section{Flow Visualization}

The system used in the experiments described here is based on the focused shadowgraphy setup shown in Fig. 2. It essentially consists of a light source, a microscope objective to expand the light beam, a second lens to produce a collimated beam going through the field of interest (e.g., a wind-tunnel test section), and a similar lens to collect the light, directing it into a photographic lens system that is coupled to a CMOS detector. The use of a telescopic lens arrangement on the imaging side allows the focusing plane of the shadowgraphy system to be moved through the depth of the test volume by changing the position of the photographic lens and camera. Thus a sharp image of any object in the viewing area can be obtained. By adjusting the focus of the lens, the sharpness of the shocks as seen by the camera can be easily adjusted; by slightly defocusing, features can be made to show up more clearly.

The projection and imaging optics are mounted on a compact and portable optical breadboard system, which can be positioned easily as two separate units on either side of the test facility (here a wind tunnel). The two separate units can be moved away for tunnel maintenance and easily put back in position, maintaining alignment. The whole arrangement measures $2438 \mathrm{~mm}$ (96 in.) from end to end, and the collimated beam section is $133 \mathrm{~mm}\left(5 \frac{1}{4} \mathrm{in}.\right)$ in diameter (determined by the diameter of the tunnel windows, the collimating and imaging lenses have a diameter of approximately $135 \mathrm{~mm}$ ). 


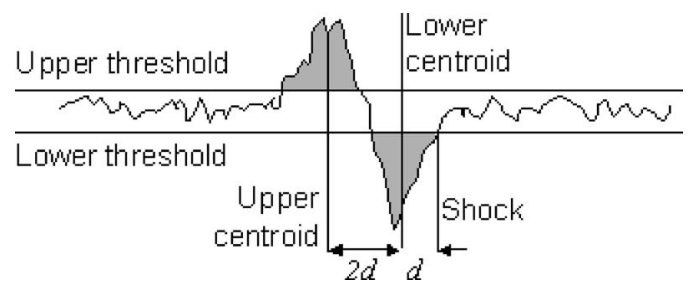

Fig. 3 Shock position identification.

The camera is mounted at $90 \mathrm{deg}$, so that the windtunnel flow is seen to be along the horizontal axis of the resulting image, from left to right.

Rather than using a discharge light source such as a mercury arc lamp, a white LED is utilized. Unlike discharge sources, the solid-state device provides a stable and repeatable intensity level, with no jittering in its exact lightemitting location. LEDs also offer long-life performance $(>100,000 \mathrm{~h})$ in a compact package and can be pulsed quickly-typical rise times being less than $100 \mathrm{~ns}$.

The selected LED (Microbeam ${ }^{\mathrm{TM}}$ Flashlight, Brookstone Company Inc., Mexico, MO, USA) comprised a blueemitting chip covered in a yellow-emitting phosphor layer. To obtain a more even light distribution a yellow filter was placed after the microscope objective in order to block the blue component of the light emitted by such LEDs.

\section{Shock Tracking}

Shadowgraphy is based on the fact that light beams that travel through a medium with a variation in refractive index, as in compressible flows around an object, will be refracted, ${ }^{1}$ bending towards the region of higher refractive index. Thus, shock waves in a flow show up as narrow bands consisting of a light and a dark area in the recording plane. In a focused shadowgraphy system the shock location can be determined as the outer edge of the dark area. This forms the basis of the high-speed shock-tracking algorithm that was developed for this study.

In the high-speed shock-tracking mode, only the last four rows of any $1280 \times 8$ frame delivered by the camera are considered. Given that 8 pixels here typically represent less than $0.5 \mathrm{~mm}$ in the imaged plane (for the $5 \frac{1}{4}$-in. viewing area), the frames are vertically integrated to give a noise-reduced one-dimensional array (neglecting shock curvature). Variations in intensity caused by uneven illumination and steady dirt on (e.g.) the windows of the wind tunnel are eliminated by subtracting an integrated strip from the same location in an image taken when there was no shock present, to produce a referenced strip. Each frame is then processed to find the positions of shocks. The resulting position data are plotted on screen, saved to disk, and outputted electronically using an 8-bit digital-to-analog converter connected to the PC parallel port.

\subsection{Shock Position}

To distinguish dark-bright variations in the referenced data strips associated with shocks from variations due to noise, upper and lower thresholds were applied (Fig. 3). To determine these threshold values, the referenced data strip is divided into ten sections containing an equal number of pixels. The minimum and maximum values are then deter- mined for each section, and the upper and lower threshold values subsequently set as the values of the third largest maximum and the third smallest minimum, respectively. The centroids of the dark and bright parts of the shock image are then calculated as

centroid $_{\text {lower }}=\frac{\sum_{x \in A}\left|I_{x}-t_{\text {lower }}\right| \cdot x}{\sum_{x \in A}\left|I_{x}-t_{\text {lower }}\right|}$,

centroid $_{\text {upper }}=\frac{\sum_{x \in B}\left|I_{x}-t_{\text {upper }}\right| \cdot x}{\sum_{x \in B}\left|I_{x}-t_{\text {upper }}\right|}$,

where $I$ is the referenced intensity, $t_{\text {lower }}$ and $t_{\text {upper }}$ the threshold values, and $x$ the pixel position. In these equations, $A$ comprises those pixels where $I<t_{\text {lower }}$, and $B$ those pixels where $I>t_{\text {upper }}$.

The calculated positions of the upper and lower threshold are then used to determine the shock position, which is defined to be a distance $d$ away from the position of the lower centroid ( $3 d$ from the upper centroid), where $d$ is half the distance between the upper and lower centroids (Fig. 3). Thus, if the upper centroid is to the left of the lower centroid (as would be the case for a bow shock generated around an object by a flow from the left), shock position can be calculated as:

shock position $=$ centroid $_{\text {lower }}+\frac{\text { centroid }_{\text {lower }}-\text { centroid }_{\text {upper }}}{2}$.

For a given number of pixels in the area considered to represent a shock, the accuracy of the calculated position is dependent on the intensity and distribution of noise within the referenced intensity data. Assuming the pixel intensities beyond the thresholds form symmetrical distributions, then the error in the calculated position is given by

error $=\frac{n \cdot(a / 2)^{2}+n \cdot(b / 2)^{2}}{\sum_{x \in A}\left|I_{x}-t_{\text {lower }}\right|+\sum_{x \in B}\left|I_{x}-t_{\text {upper }}\right|}$,

where $n$ is the maximum noise intensity, and $a$ and $b$ are the number of pixels in $A$ and $B$, respectively.

\section{Numerical Simulation}

For comparison, axisymmetric numerical simulations were made using the high-accuracy NTS Navier-Stokes solver, ${ }^{11,12}$ which is capable of solving flow conditions with a wide range of Mach numbers. For the nonlinear spatial derivatives Roe's ${ }^{13}$ flux difference splitting method is used. For this, underlying interpolations can be up to fifth-order accuracy. The solution is marched forward in time using a second-order implicit backward differentiation.

The code is run in parallel processor mode, and the simulation is performed on a specially formed grid of approximately 400,000 nodes to take into account the coneshaped nozzle. Because of the low geometrical complexity, the grid is generated algebraically. The grid consists of two blocks: an inner and an outer block. To resolve the more intricate flow features around the pulsating jet region, an especially fine inner block grid is used, to avoid a singularity at the axis. The inner block's radial extent is in the region of 0.0001 to $0.005 \mathrm{~m}$. The simulation is run in un- 


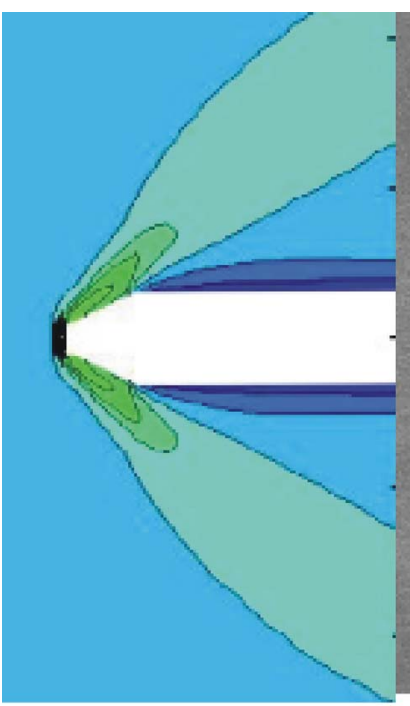

(a)

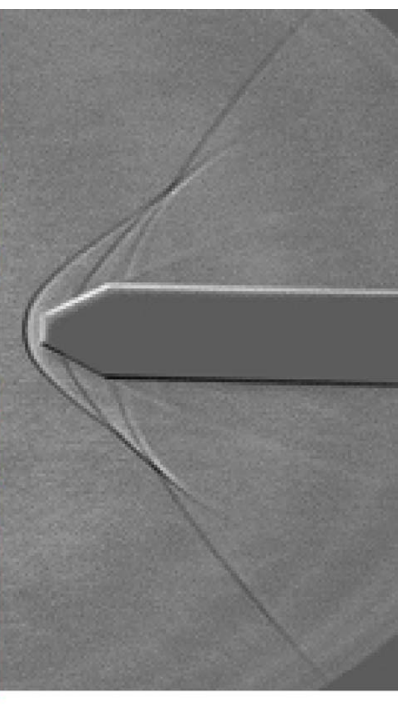

(b)
Fig. 4 Comparison for $M_{t}=2$, no jet: (a) density plot of center-plane Navier-Stokes solution (b) shadowgraphy, still view.

steady mode, needing around 10 subiterations per time step. The real time step $(t)$ is related to a nondimensional time step $t^{*}=t v_{0} / l_{0}$, where $v_{0}$ and $l_{0}$ represent the velocity and length scales, respectively. Using a nondimensional time step of $t^{*}=0.5$ is found to be successful. All simulations are run in parallel OpenMP mode.

In the present study, free jet nozzle flows are not explicitly solved, but are treated by prescribing a suitable velocity profile as an inlet boundary condition. A power-law velocity profile is specified at the jet outlet; the Mach numbers of the jet flow and the tunnel flow can each be at a maximum of 2.0. A sinusoidal disturbance is artificially added to the assumed mean velocity profile at the nozzle exit and normalized by the centerline inlet velocity. Near to the jet region a fine grid is used so as to resolve detailed flow structures. The results are circumferentially averaged.

\section{Results}

All results shown here are normalized after subtracting a no-flow background image, minimizing effects of uneven illumination and dirt.

\subsection{Still View}

Figure 4(b) shows the still view when the tunnel is operating at Mach $M=2.06$, without a through-nozzle flow, while Fig. 4(a) shows the Navier-Stokes result. A complex shock pattern is seen. Note that the numerical result shows the density field in the center plane, whereas the shadowgraph is the result of light bending integrated over the full width of the flow field. Although due to this the shocks cannot be directly seen, the expansion wave at the junction of the cone with the main body can be identified. Furthermore, the bend in the bow shock seen in the shadowgraph is reproduced in the density field obtained numerically.

Figure 5(b) shows the still view with tunnel flow and steady through-nozzle flow (at total jet pressure $P_{j}$ $=3.7$ bar, $M_{j}=1.5$ ), while the Navier-Stokes result is shown

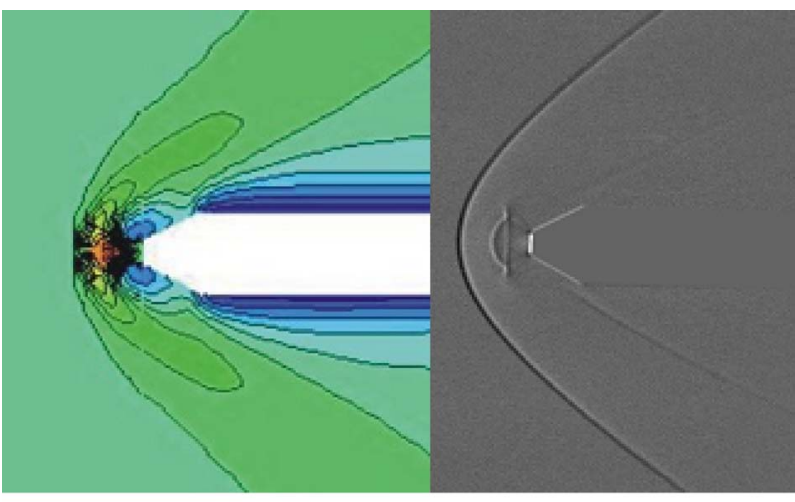

(a)

(b)

Fig. 5 Comparison: (a) density plot of Navier-Stokes solution $\left(M_{t}=2, M_{j}=2\right)$; (b) shadowgraph, still mode, $M_{t}=2.06, M_{j}=1.5$.

in Fig. 5(a) (note that the jet Mach number here is somewhat higher, at 2.0). The multiple shocks can be seen to reduce to a single strong bow shock at an increased standoff distance from the nozzle, and another shock system with inverted density gradient is seen in front of the nozzle. Again, good correspondence is found between the shapes and positions of both the bow shock and the expansion wave. Also, the numerical result gives an indication for the causes for the particular shock structure found between the bow shock and the nozzle exit. Behind the bow shock a strong compression takes place, resulting in a small highdensity region between the jet and the bow shock, apparently bordered by a high density gradient.

\subsection{Spatial-Temporal Streak View}

When the through-nozzle flow-and therefore the shock position-is steady, the spatial-temporal streak view is identical to the still view. When the through-nozzle jet flow is modulated, however, a clear modulation of the shocks is shown in the streak view (Fig. 6). Figure 6(a) and 6(b) show the spatial-temporal streak view when the throughnozzle flow is modulated between 1.7 and 3.7 bar at 80 and $550 \mathrm{~Hz}$, respectively.

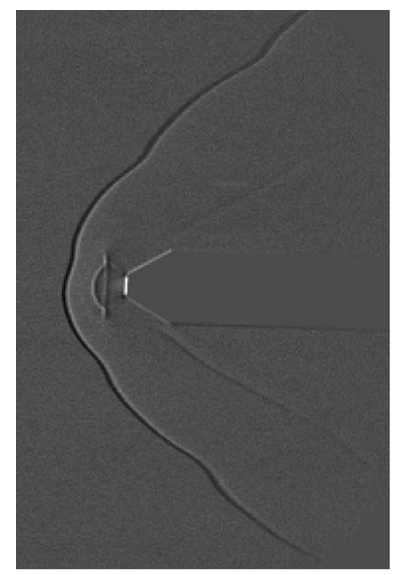

(a)

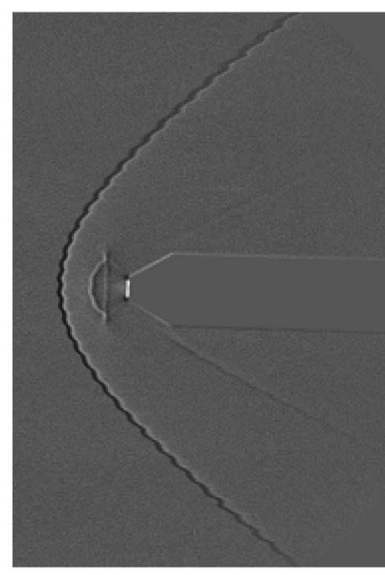

(b)
Fig. 6 Shadowgraphy result obtained in spatial-temporal mode. $M_{t}=2.06, M_{j} \approx 0.9$ to $1.5:$ (a) $f \approx 80 \mathrm{~Hz}$, (b) $f \approx 550 \mathrm{~Hz}$. 


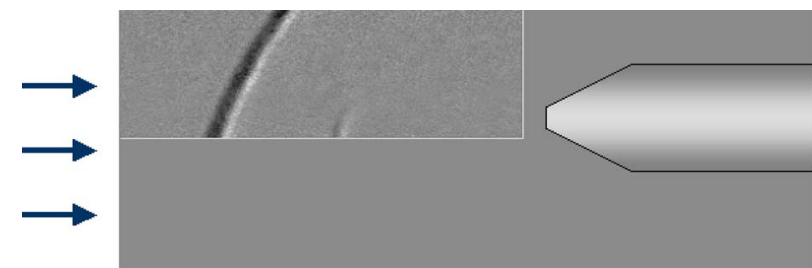

Video 1. Shadowgraphy visualization of shock oscillation, taken at $200 \mathrm{frames} / \mathrm{s}$ (MPEG, $860 \mathrm{~KB}$ ).

[URL: http://dx.doi.org/10.1117/1.2992621.1].

Although the images obtained in this mode are not straightforward to interpret, they contain useful information that is not present in those obtained in the more conventional still mode.

Firstly, the image contains spatial information, although slightly complex to extract. The wobbly image of the bow shock gives a misleading impression of irregularly shaped shock waves, for the instantaneous still views of the same flow conditions show images similar to that in Figure 5(b), i.e., the shocks remain smooth. Therefore, it can be concluded that due to the jet modulation, the bow shock is moved to and from the jet nozzle. Thus, the irregularity indicates movement, not deformation. This is confirmed by the video still shown in Video 1, where a windowed view of the shock oscillation is shown, taken at $200 \mathrm{frames} / \mathrm{s}$, as well as by the numerical result for the oscillating flow shown in Video 2. In both cases the images of the bow shock are affected the most, as shown by the clear periodicity in Fig. 6(a) and 6(b). The effect also shows up in the shock closest to the bow shock as well as in the ones originating close to the cone-cylinder transition. The diverging shocks going from the nozzle exit and the vertical shock at their end points (Mach disk) do not show signs of unsteadiness, indicating they remain in position.

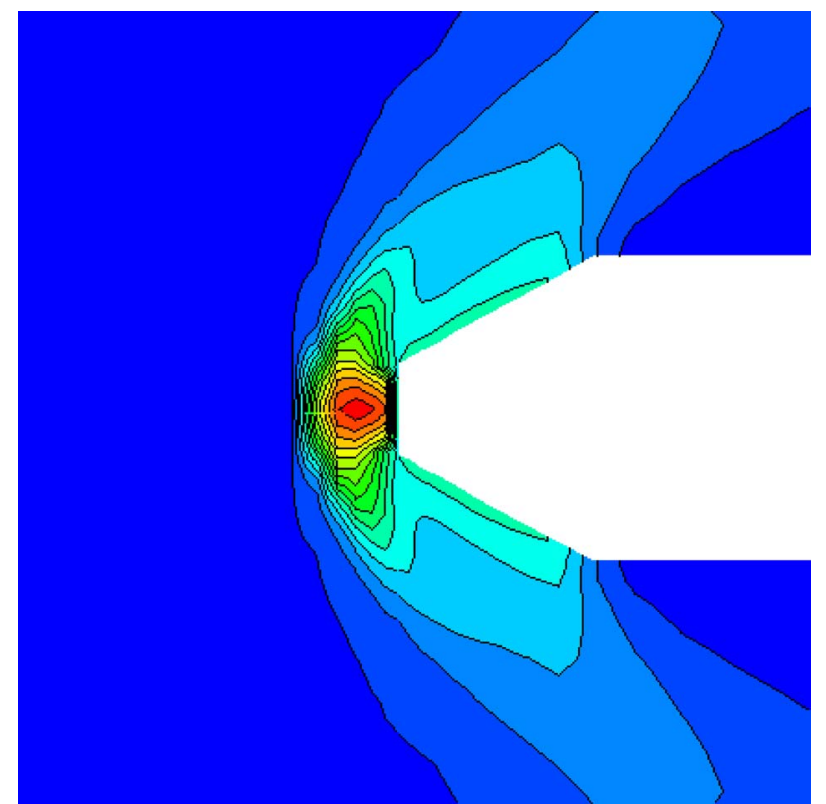

Video 2. Numerical simulation of shock oscillation (MPEG, 986 KB). [URL: http://dx.doi.org/10.1117/1.2992621.2].

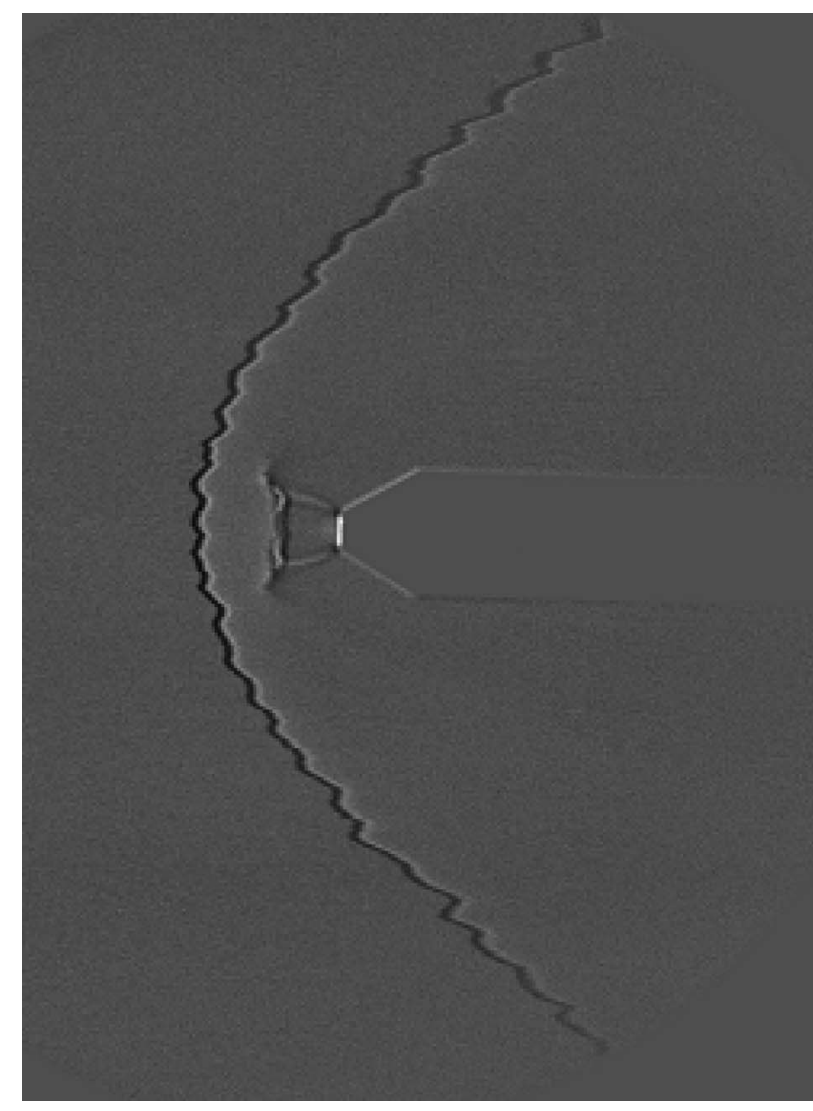

Fig. 7 Spatial-temporal view of unstable oscillation (jet nozzle pressure $>3.7$ bar)

Secondly, by analyzing the modulations seen in spatialtemporal images such as those shown in Fig. 6, the frequency of the movement can be determined. In Fig. 6(a) about 6 or 7 oscillations are seen in the bow shock; at a framing rate of $12 \mathrm{frames} / \mathrm{s}$ this would correspond to a frequency of $[(1 / 12) / 6.5]^{-1} \approx 78 \mathrm{~Hz}$. For the flow in Fig. 6(b), where about 44 oscillation periods are seen, the estimate is $\approx 530 \mathrm{~Hz}$. Note that the modulation frequency could only be set indirectly, and the resulting frequency could not be determined accurately from the valve motor. From the shock-tracking experiments described later it was found that the set frequency was typically about $5 \%$ higher than the frequency determined from the tracking measurements. Thus, given the framing rate, a reasonable estimate of the oscillation period in the flow can be determined from the number of oscillations in the image.

Increasing the total pressure for the modulated nozzle flow to above 3.7 bar results in an unstable wavering, as shown in Fig. 7, while the still view is still similar to Fig. $5(\mathrm{~b})$. In this case, a clear disturbance is seen not only in the bow shock and the hemispherical one closest to it, but also in all other shocks. The spatial-temporal streak view shown in Fig. 7 clearly depicts the irregular motion of the shock wave in contrast to the images shown in Figs. 6(a) and 6(b), whereas an instantaneous view such as Fig. 5(b) offers no such information. The still images and spatial-temporal streak view images are complementary, suggesting in this 


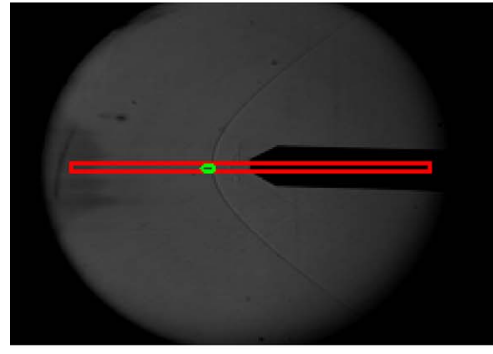

(a)

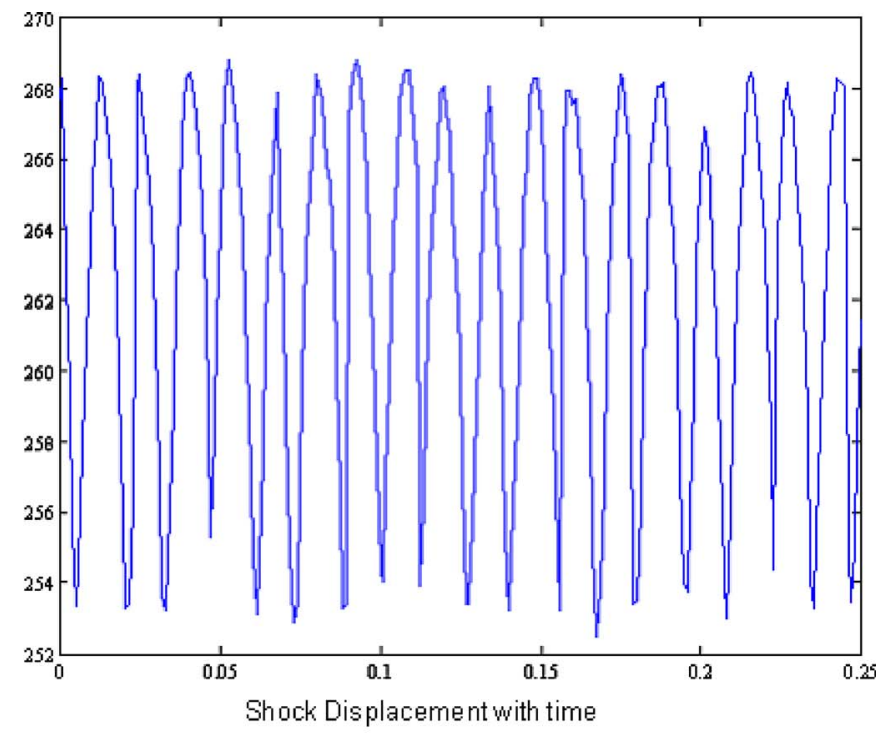

(b)

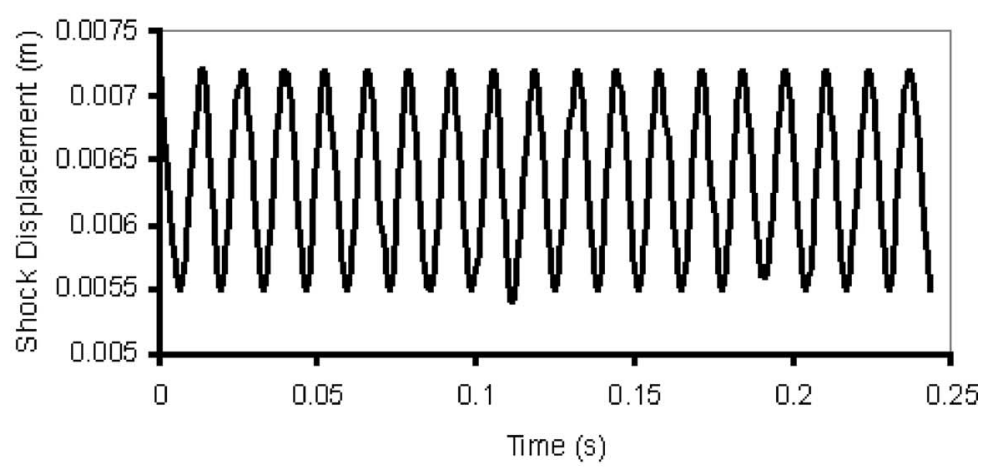

(c)

Fig. 8 Shock tracking: shock position versus time for 76-Hz oscillation: (a) shock position; (b) shadowgraph tracking: amplitude 16 pixels, $\approx 2 \mathrm{~mm},(\mathrm{c})$ Navier-Stokes result: amplitude $\approx 1.7 \mathrm{~mm}$.

case that the bow shock remains smooth and is displaced in its entirety (horizontally in the images), but not with a regular oscillation.

\subsection{Tracking View}

Shock-tracking results for the modulation at $80 \mathrm{~Hz}$ nominally are shown in Fig. 8. Figure 8(b) shows a typical position plot, where the position was measured along the central axis of the nozzle [as shown in Fig. 8(a)], showing an actual shock modulation frequency of $76 \mathrm{~Hz}$. The numerical result at this oscillation frequency [Fig. 8(c)] indicates an amplitude of shock movement of approximately $1.7 \mathrm{~mm}$, which corresponds very well with the tracking result of 16 pixels, corresponding to $\approx 2 \mathrm{~mm}$.

The amplitude of shock motion is found to decrease with increasing excitation oscillation frequency, similar to that found by Doerffer et al. ${ }^{14}$ in their studies of forced shock oscillations.

Examination of the pixel intensities at any one instant shows that the bright and dark areas that form the shock are very close to being symmetrical distributions. Thus, the position error may be calculated using the equation described previously. For the data shown, this equates to approximately 0.6 pixels, or $4 \%$ of the total oscillation amplitude.

\subsection{Shock Angle Measurement}

To demonstrate the use of the system for monitoring shock angle (e.g., to facilitate the study of unsteady oblique shock waves), two daisy-chained cameras were run in tracking mode simultaneously. The cameras were mounted either side of a beamsplitter, placed after the imaging lens, and positioned to view the same area [Fig. 9(a)]. The gains were adjusted to compensate for the unequal reflectance and transmittance of the beamsplitter. When running in tracking mode, the sensor subwindow regions were set such that one camera was viewing an area above the other [Fig. 9(b)]. The shock angle was then calculated from the resulting two positions. For the bow shock in the flow studied here, an angle variation is found between 36 and 38 deg to the horizontal, corresponding fairly well to the 35.2 to $37.8 \mathrm{deg}$ found from the Navier-Stokes simulations.

\section{Discussion and Conclusions}

The use of a low-cost CMOS sensor to achieve both fullfield-of-view megapixel flow visualizations and high-speed 

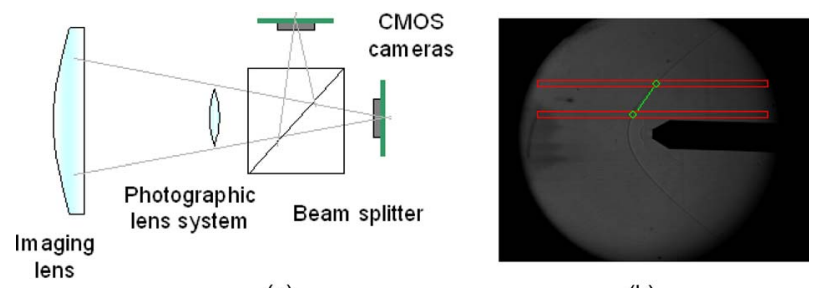

(a)

(b)

Fig. 9 Camera configuration for shock angle measurement: (a) imaging part of experimental setup, (b) total view for both cameras with viewing windows for the two cameras in tracking mode indicated by red rectangles (top rectangle, camera 1; bottom rectangle, camera 2). (Color online only.)

shock position tracking has been demonstrated in a lowcost digital focused shadowgraphy system tested on unsteady supersonic flow fields. The system uses three current advances in technology: a robust, low-cost, ultrabright white-light LED as a light source, enabling microsecond freeze-frame imaging of constant illumination quality; a CMOS camera for digital recording, allowing direct control over the size of the captured image, and making it possible to trade off image size for image frame rate; and FireWire communications technology to transmit the images directly to a computer, which can then be used as input for a shockstabilizing control system.

The system allows data to be recorded in three basic modes of operation: a still full-field-of-view freeze-frame mode (with the current system at $1280 \times 1024$-pixel resolution and 2 frames/s), a windowed tracking mode (980 frames/s at $1280 \times 8$ pixels), and a continuous fullfield-of-view mode $(1280 \times 1024,12$ frames/s). This last mode is based on the fundamental mode of operation of CMOS cameras and allows a new type of visualization to be obtained: spatial-temporal streak imaging. Although the images obtained in this mode are by no means trivial to interpret, they do help in the immediate identification of regions of instability as well as giving information on instability frequencies and amplitude.

Expanding the system with a second identical camera looking at the same flow region using a beamsplitter allows high-speed simultaneous tracking of features at different locations in the flow. Thus, e.g., the local angle of shock waves can be determined and monitored for changes.

Because the CMOS camera uses FireWire connection, image processing can be performed immediately in the host computer. Thus, the system presented here can track the position of a flow feature at a rate of $980 \mathrm{~Hz}$ (determined by camera frame rate). The images reach the $\mathrm{PC} \approx 250 \mu \mathrm{s}$ after the event, and the position is then calculated in less than $1 \mu \mathrm{s}$. A real-time 8-bit electronic output can then be sent via a parallel port with less than $10-\mu$ s lag. Thus, the position information can be sent directly to a flow control system, with a delay $<300 \mu$ s, allowing immediate feedback as input for a flow adjustment and controlling device, e.g., for controlling the shock position in a supersonic inlet.

The constructed system has low cost (£1000 for camera plus light source) and can be generalized and miniaturized in several ways. Thus, it has the potential to be used, e.g., for making an intrapassage transonic rotor shock measurement to provide active control within a gas turbine engine.

The system is highly versatile and suitable to a wide range of applications, including shock control, study of forced shock oscillations, ${ }^{14}$ and aerodynamic heating reduction. ${ }^{6}$

The results obtained with the shadowgraphy visualization and tracking system are confirmed and clarified by the numerical results. Good correspondence is found for shock positions and angle oscillation.

\section{Acknowledgments}

The Warwick OEL team wish to thank Prof. A. Epstein for inviting them to participate in the research program at MIT. Part of this work was supported through a European Community Individual Marie Curie Fellowship "An Intelligent Optical Diagnostic for Combustion," contract No. HPMFCT-2000-00997, by the UK Engineering \& Physical Sciences Research Council (EPSRC), by the Warwick IMRC (RESCM9002), and by the U.S. Defense Advanced Research Project Agency (DARPA). Furthermore, the authors acknowledge the advice of Prof. Carpenter of Warwick University on flow conditions and the invaluable assistance of J. Letendre, J. Costa, V. Dubrowski, J. Head, and D. Allaire of the Gas Turbine Laboratory of MIT in performing the experiments described in this paper.

\section{References}

1. W. Merzkirch, Flow Visualization, 2nd ed., Academic Press (1987).

2. G. S. Settles, "High-speed imaging of shock waves, explosions and gunshots," Am. Sci. 94(1), 22 (2006)

3. S. R. Donohoe and W. J. Bannink, "Surface reflective visualizations of shock-wave/vortex interactions above a delta wing," AIAA J. 35(10), 1568-1573 (1997).

4. B. H. Timmerman, "Holographic interferometric tomography for unsteady compressible flows," PhD Thesis, Delft Univ. of Technology, Eburon P\&L, Delft, The Netherlands (1997).

5. A. Ben-Yakar and R. K. Hanson, "Ultra-fast-framing schlieren system for studies of the time evolution of jets in supersonic crossflows," Exp. Fluids 32, 652-666 (2002).

6. I. Fenercioglu and K. B. Yuceil, "Determination of shock oscillation character by instantaneous imaging of bow shock wave caused by a projectile," presented at 12th Int. Symp. on Flow Visualization, September 10-14, 2006, Göttingen, Germany (2006).

7. T. Fukuchi and K. Nemoto, "High-speed shadowgraphy and interferometry using an acousto-optic laser deflector," presented at Ultrafast $X$-Ray Detectors, High-Speed Imaging, and Applications, Proc. SPIE 5920, 59200R (2005).

8. J. R. Gord, C. Tyler, K. D. Grinstead, G. J. Fiechtner, M. J. Cochran, and J. R. Frus, "Imaging strategies for the study of gas turbine spark ignition," in Optical Diagnostics for Fluids/Heat/Combustion and Photomechanics for Solids, Proc. SPIE 3783, 352-361 (1998).

9. R. J. H. Klein-Douwel, P. J. M. Frijters, L. M. T. Somers, W. A. de Boer, and R. S. G. Baert, "Macroscopic diesel fuel spray shadowgraphy using high speed digital imaging in a high pressure cell," Fuel 86, 1994-2007 (2007).

10. T. Homae, T. Moritoh, K. Ooya, K. G. Nakamura, and K. Kondo, "A simple fiber-optic pin for detecting a shock-wave front," Rev. Sci. Instrum. 71(11), 4192-4194 (2000).

11. P. G. Tucker, "Novel MILES computations for jet flows and noise," Int. J. Heat Fluid Flow 25(4), 625-635 (2004).

12. M. L. Shur, P. R. Spalart, M. K. Strelets, and A. K. Travin, "Towards the prediction of noise from jet engines," Int. J. Heat Fluid Flow 24, 551-561 (2003).

13. P. L. Roe, "Approximate Riemann solvers, parameter vectors and difference schemes," J. Comput. Phys. 46, 357-378 (1981).

14. P. Doerffer, O. Szulc, and F. Magagnato, "Shock wave-boundary layer interaction in forced shock oscillations," J. Therm. Sci. 12(1), 10-15 (2003).

Biographies and photographs of authors not available. 